\title{
Prediction of Tumor Recurrence in Patients with Non- Gastric Gastrointestinal Stromal Tumors Following Resection according to the Modified National Institutes of Health Criteria
}

\author{
Seung Hyeon Jang*, Ji Eun Kwon*, Jee Hyun Kim, June Young Lee, Sang Gyun Kim, Joo Sung Kim, \\ Hyun Chae Jung, Jong Pil Im \\ Department of Internal Medicine and Liver Research Institute, Seoul National University College of Medicine, Seoul, Korea \\ *Both authors contributed equally to this work.
}

Background/Aims: Few studies have investigated the prognosis of non-gastric gastrointestinal stromal tumors (GISTs) under the modified National Institutes of Health (NIH) consensus criteria in Korea. This study aims to clarify the clinical usefulness of the modified NIH criteria for risk stratification. Methods: From January 2000 through October 2012, 88 patients who underwent curative resection for primary GISTs were included in this study. The enrolled patients were stratified to predict recurrence by the original NIH criteria and modified NIH criteria. Results: In all, 88 patients had non-gastric GISTs, including 82 and 6 patients with GISTs of the small intestine and colorectum, respectively. The mean age was $57.3 \pm 13.0$ years, and the median follow-up duration was 3.40 years (range, $0.02-12.76$ years). All patients who were placed in the intermediate-risk category according to the original NIH criteria were reclassified into the high-risk category according to the modified NIH criteria. Therefore, the proportion of cases in the intermediate-risk category declined to $0.0 \%$ from $25.0 \%$ (22/88), and the proportion of cases in the high-risk category increased to $43.2 \%$ (38/88) from 18.2\% (16/88) under the modified NIH criteria. Among the 22 reclassified patients, $6(27.3 \%)$ suffered a recurrence during the observational period, and the recurrence rate of high-risk category patients was 36.8\% (14/38). Conclusions: Patients in the high-risk category according to the modified NIH criteria had a high GIST recurrence rate. Therefore, the modified NIH criteria are clinically useful in selecting patients who need imatinib adjuvant chemotherapy after curative surgical resection. (Intest Res 2014;12:229-235)

Key Words: Gastrointestinal stromal tumors; Prognosis; Recurrence; National Institutes of Health (U.S.)

\section{INTRODUCTION}

Gastrointestinal stromal tumors (GISTs) are the most

Received November 18, 2013. Revised February 4, 2014.

Accepted February 4, 2014.

Correspondence to Jong Pil Im, Department of Internal Medicine and Liver Research Institute, Seoul National University College of Medicine, 101 Daehak-ro, Jongno-gu, Seoul 110-744, Korea. Tel: +82-2-740-8112, Fax: +82-2-743-6701, E-mail:.jp-im@hanmail.net

Financial support: This study was supported by grant No. 04-2009-4659 from the Seoul National University Hospital research fund. Conflict of interest: None. common mesenchymal tumors of the gastrointestinal (GI) tract and typically arise from the interstitial cells of Cajal. Primary GISTs arise most commonly in the stomach (50-70\%) or small bowel (20-30\%), but may arise anywhere in the GI tract or rarely outside the GI tract as primary tumors. ${ }^{1}$ The positivity for the c-kit (CD117) receptor tyrosine kinase, observed in approximately 95\% of GISTs, is the molecular hallmark of GISTs. Widespread use of immunohistochemical staining for c-kit has now enabled accurate diagnosis. ${ }^{2}$

Surgical resection has been the gold standard for cure in the setting of localized GISTs. Although tumor recurrence

๑ Copyright 2014. Korean Association for the Study of Intestinal Diseases. All rights reserved.

This is an Open Access article distributed under the terms of the Creative Commons Attribution Non-Commercial License (http://creativecommons.org/licenses/by-nc/3.0)

which permits unrestricted non-commercial use, distribution, and reproduction in any medium, provided the original work is properly cited. 
Table 1. Risk Stratification of Primary Gastrointestinal Stromal Tumors (GISTs) under the National Institutes of Health (NIH) Criteria and Modified NIH Criteria

\begin{tabular}{|c|c|c|c|c|c|}
\hline \multicolumn{3}{|c|}{ Tumor parameter } & \multirow{2}{*}{ NIH criteria } & \multicolumn{2}{|c|}{ Modified NIH criteria } \\
\hline Mitotic count & Tumor size & Tumor rupture* & & Gastric & Non-gastric \\
\hline \multirow[t]{4}{*}{$\leq 5 / 50 \mathrm{HPF}$} & $\leq 2 \mathrm{~cm}$ & No & Very low & Very low & Very low \\
\hline & $>2, \leq 5 \mathrm{~cm}$ & No & Low & Low & Low \\
\hline & $>5, \leq 10 \mathrm{~cm}$ & No & Intermediate & Intermediate & High \\
\hline & $>10 \mathrm{~cm}$ & No & High & High & High \\
\hline \multirow[t]{4}{*}{ 6-10/50 HPF } & $\leq 2 \mathrm{~cm}$ & No & Intermediate & Intermediate & Intermediate \\
\hline & $>2, \leq 5 \mathrm{~cm}$ & No & Intermediate & Intermediate & High \\
\hline & $>5_{1} \leq 10 \mathrm{~cm}$ & No & High & High & High \\
\hline & $>10 \mathrm{~cm}$ & No & High & High & High \\
\hline$>10 / 50 \mathrm{HPF}$ & Any & No & High & High & High \\
\hline Any* & Any* & Yes $^{*}$ & $-{ }^{*}$ & High* $^{*}$ & $\mathrm{High}^{*}$ \\
\hline
\end{tabular}

${ }^{*}$ The presence of tumor rupture was added to the modified $\mathrm{NIH}$ criteria as a poor prognostic factor.

HPF, high-power field.

is common and usually occurs in the liver and peritoneum, accurate assessment of the recurrence risk after curative resection is difficult. Many studies have focused on stratifying GISTs into prognostic categories based on different parameters; it is generally agreed that tumor size and mitotic count are important prognostic factors at all sites. Therefore, the National Institutes of Health (NIH) consensus criteria, which are based on tumor size and mitotic index, have been used to assess recurrence risk and to predict prognosis. ${ }^{3}$ In a large study with long-term follow-up, the location of the primary tumor was an important prognostic factor. In that study, GISTs of the stomach showed a much lower rate of aggressive behavior than GISTs of the small bowel that were similar in size and/or mitotic rate. ${ }^{4-6}$ Furthermore, tumor rupture became known as an independent risk factor that negatively impacted recurrence-free survival (RFS). ${ }^{7,8}$ Accordingly, a modification of the NIH criteria was proposed to distinguish prognosis after GIST resection (Table 1). ${ }^{3.9}$

The success of imatinib (Glivec ${ }^{\circledR}$, Novartis Korea, Seoul, Korea) in the setting of advanced GIST prompted interest in its use in the adjuvant setting after complete resection of a primary tumor. ${ }^{10-12}$ Recent studies showed that adjuvant imatinib therapy reduced the recurrence risk after curative resection of localized GISTs. ${ }^{13,14}$ However, improvement of RFS was marginal in patients with tumors $<10 \mathrm{~cm}$, although the recurrence risk was almost halved in patients with a primary tumor $\geq 10 \mathrm{~cm}^{15}$ Therefore, the need for accurate risk stratification has become increasingly important, because it is crucial for selecting patients who are most likely to benefit from adjuvant imatinib therapy. ${ }^{16}$ Recent studies showed that the modified NIH criteria were the best classification to identify a single high-risk group for consideration of adjuvant therapy. ${ }^{17-19}$ However, most of these studies were conducted in Western nations, and studies about the risk classification systems to predict prognosis after surgical resection in East Asian patients with GIST are scarce. Therefore, we performed this study to clarify the clinical usefulness of the modified NIH criteria, as compared with the original NIH criteria, in predicting recurrence of GISTs in the small intestine and colorectum after curative resection in Korea.

\section{METHODS}

\section{Patients}

The patients who underwent curative surgery for primary GISTs of the small intestine and colorectum without metastasis between January 2000 and October 2012 at Seoul National University Hospital were enrolled. Those who received adjuvant chemotherapy including imatinib after surgery were excluded to observe the natural course of the tumor. Their clinical data regarding age, sex, tumor site, dates of surgery and the last follow-up, and pathologic reports including tumor size and mitotic rates were collected and analyzed retrospectively. The histological diagnosis of the surgical specimen was based on H\&E and immunohistochemical staining for c-kit (CD117), CD34, S-100 protein, and smooth muscle actin. Patients were diagnosed with GISTs and included in this study if they had c-kit (CD117) and CD34-positive tumors with typical histologic patterns of 
spindle or epithelioid cell types. This study was approved by the institutional review board.

\section{Risk Classification and Follow-up}

The original NIH criteria stratified tumors into the very low-, low-, intermediate-, and high-risk categories, based on tumor size and mitotic count. After modification of the NIH criteria, the site of the primary tumor and tumor rupture were included as prognostic factors (Table 1). The patients were stratified into one of the four categories according to the NIH criteria and modified NIH criteria, and the changes of each risk category were analyzed.

Patients were followed to detect recurrence with endoscopy and/or an imaging modality including abdominal/pelvic CT on a regular basis of 6 to 12 months. RFS was defined from the date of radical surgery to the date of tumor recurrence.

\section{Prognostic Factors}

Tumor size and mitotic count are important prognostic factors at all sites. The site of origin, tumor rupture, and several histologic features, including serosal involvement and necrosis, are also known as prognostic factors. These factors were evaluated as potentially significant prognostic factors for tumor recurrence.

\section{Statistical Analysis}

Continuous variables were expressed as means $\pm S D$ or median with range. Intergroup comparison of clinicopathologi-

Table 2. Clinicopathological Characteristics of the Present Study Population according to the Tumor Site

\begin{tabular}{lc}
\hline \multicolumn{1}{c}{ Variables } & $\mathbf{n}=\mathbf{8 8}$ \\
\hline Age $(\mathrm{yr})$ & $57.5 \pm 13.0$ \\
Male & $46(52.3)$ \\
Tumor size $(\mathrm{cm})$ & $5.0 \pm 3.8$ \\
$\quad \leq 5$ & $62(69.6)$ \\
$>5$ & $26(30.4)$ \\
Mitotic count (HPF) & $7.7 \pm 15.9$ \\
$\quad \leq 5 / 50$ & $65(73.0)$ \\
$>5 / 50$ & $24(37.0)$ \\
Median follow-up (yr, range) & $3.40(0.02-12.76)$ \\
\hline
\end{tabular}

Values are presented as mean \pm SD or $n(\%)$.

HPF, high-power field. cal features was done with the Student's $t$-test. Categorical variables were compared with the Pearson's chi-square test. RFS was calculated with the Kaplan-Meier method, and the difference among groups was determined with the log-rank test. For the prognostic factors evaluation, each variable was assessed by performing univariate analysis. The variables with statistical significance were included in a multivariate analysis using the Cox regression hazard model. Results were considered statistically significant for $P$-values $<0.05$. Statistical analysis was performed with SPSS for Windows (version 18.0; SPSS Inc., Chicago, IL, USA).

\section{RESULTS}

\section{Clinicopathological Characteristics of the Study Population}

In total, 88 patients, 82 and 6 with GISTs of the small intestine and colorectum, respectively, were included in this study (Table 2). The mean age was $57.3 \pm 13.0$ years, and 46 patients (52.3\%) were men. The median follow-up duration was 3.40 years (range, $0.02-12.76$ years). Segmental resection with end-to-end anastomosis was the most common surgical procedure. The mean tumor size was $5.0 \pm 3.8 \mathrm{~cm}$ and the mean mitotic count was $5.1 \pm 8.9$ per 50 high-power fields (HPF).

\section{Risk Stratification under the NIH and Modified NIH Criteria}

Among the 88 patients, 5 (5.7\%), 45 (51.1\%), 22 (25.0\%), and $16(18.2 \%)$ were categorized in the very low-, low-, moderate-, and high-risk groups, respectively, according to the NIH criteria (Table 3). All patients who were placed in the intermediate-risk category according to the original NIH criteria were reclassified into the high-risk category according to the modified NIH criteria based on the location

Table 3. Risk Stratification according to the National Institutes of Health (NIH) Criteria and Modified NIH Criteria and Recurrence in the Subgroup under the NIH and Modified NIH Criteria

\begin{tabular}{lccc}
\hline \multirow{2}{*}{$\begin{array}{c}\text { Risk } \\
\text { stratification }\end{array}$} & n=88 & Recurrence \\
\cline { 2 - 2 } Very low & $5(5.7) / 5(5.7)$ & NIH/Modified NIH \\
Low & $45(51.1) / 45(51.1)$ & $0 / 0$ \\
Intermediate & $22(25.0) / 0(0.0)$ & $4 / 4$ \\
High & $16(18.2) / 38(43.2)$ & $6 / 0$ \\
\hline
\end{tabular}

Values are presented as mean \pm SD or $n(\%)$. 
of the primary tumors. Therefore, the proportion of cases in the intermediate-risk category declined to $0.0 \%$ from $25.0 \%$ (22/88), and the proportion of cases in the high-risk category increased to $43.2 \%(38 / 88)$ from $18.2 \%$ (16/88) under the modified NIH criteria.

\section{Results of Follow-up}

Follow-up data were obtained in all patients to death or the date of data analysis. Eighteen patients (20.5\%) had a recurrence, and the most frequent sites were the liver and

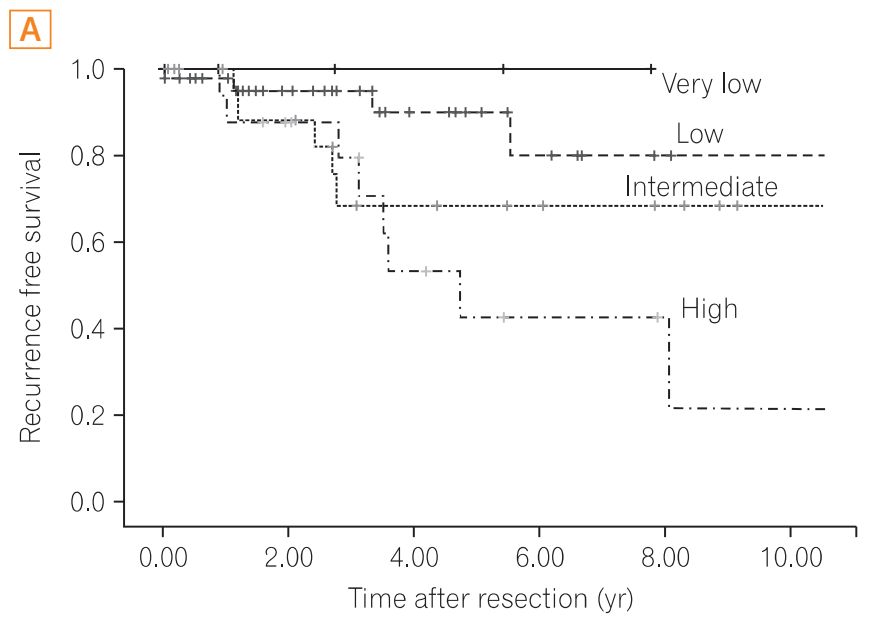

peritoneum. The Kaplan-Meier curves of RFS in the very low- and low-risk groups under both risk stratification criteria were identical (Fig. 1). Only 4 patients in the low-risk category experienced tumor recurrence. However, all of the intermediate-risk group patients of the original NIH criteria were reclassified to the high-risk group under the modified NIH criteria. Among the 22 reclassified patients from the intermediate-risk to high-risk category, 6 (27.3\%) suffered a recurrence during the observational period. Thus, recurrence developed in $14(36.8 \%)$ of 38 patients in the highrisk category (Fig. 2). The high-risk category according to

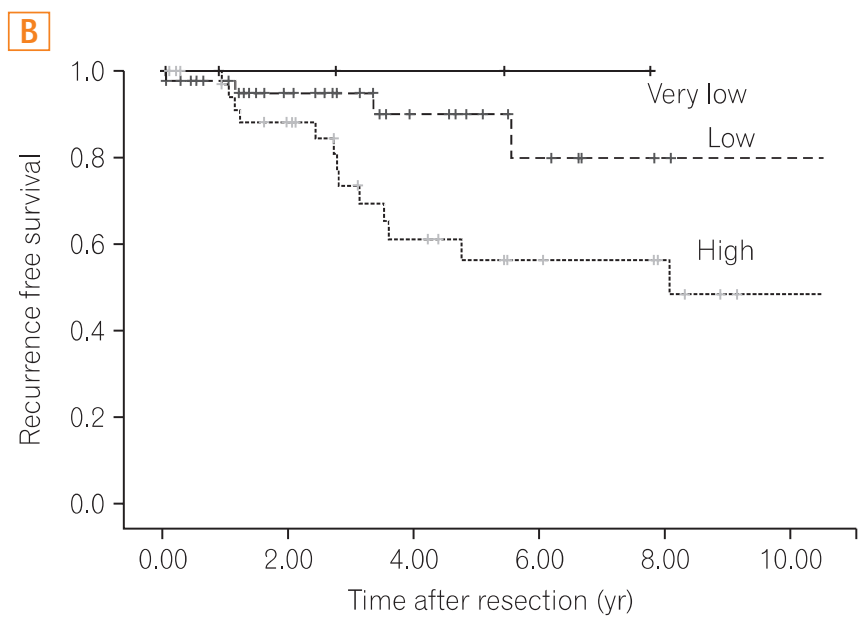

Fig. 1. Recurrence-free survival in non-gastric gastrointestinal stromal tumor (GIST) according to (A) the National Institutes of Health (NIH) criteria and (B) the modified NIH criteria. Under the modified NIH criteria, all patients with non-gastric GISTs who were placed in the intermediate-risk category according to the original $\mathrm{NIH}$ criteria were reclassified into the high-risk category. There was no difference in the very low- and low-risk categories between the original and modified NIH criteria.

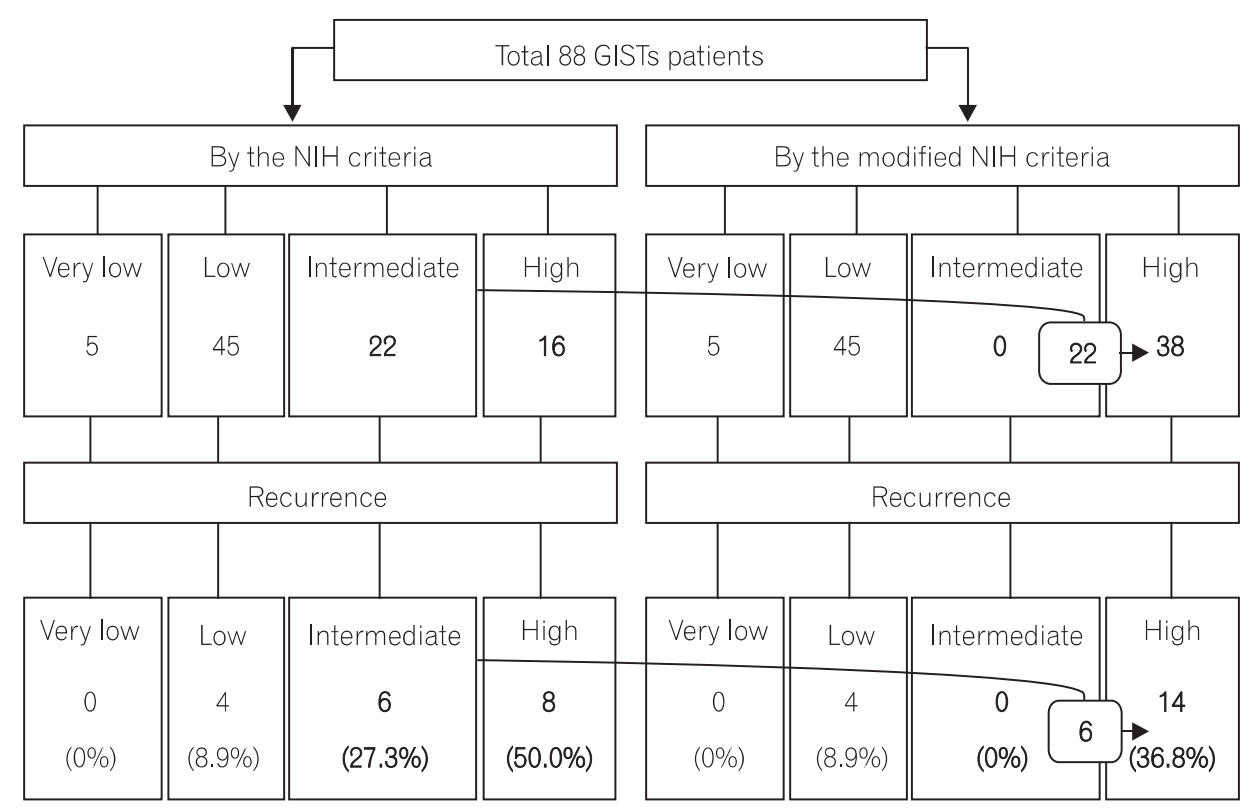

Fig. 2. Risk stratification according to the National Institutes of Health (NIH) and modified NIH criteria. Twenty-two patients with intermediate risk under the original $\mathrm{NIH}$ criteria were reclassified into the highrisk category by the modified NIH criteria. Among the 22 reclassified patients, 6 patients experienced tumor recurrence. GISTs, gastrointestinal stromal tumors. 
Table 4. Prediction Factors for Recurrence-Free Survival

\begin{tabular}{|c|c|c|c|c|c|c|c|}
\hline \multirow{2}{*}{\multicolumn{2}{|c|}{ Prognostic factor }} & \multirow{3}{*}{$\begin{array}{c}\text { n (\%) } \\
82(93.2)\end{array}$} & \multirow{3}{*}{$\begin{array}{c}\text { 5-yr RFS, \% } \\
73.9\end{array}$} & \multirow{3}{*}{$\begin{array}{c}\text { Univariate* }^{*} \\
\boldsymbol{P} \text {-value } \\
0.491\end{array}$} & \multicolumn{3}{|c|}{ Multivariate $^{+}$} \\
\hline & & & & & \multirow[t]{2}{*}{ HR } & \multirow[t]{2}{*}{$95 \% \mathrm{Cl}$} & \multirow[t]{2}{*}{$P$-value } \\
\hline Primary site & Small bowel & & & & & & \\
\hline & Colorectum & $6(6.8)$ & 50.0 & & & & \\
\hline \multirow[t]{2}{*}{ Size } & $\leq 5 \mathrm{~cm}$ & $62(70.5)$ & 86.8 & 0.05 & & & 0.19 \\
\hline & $>5 \mathrm{~cm}$ & $26(29.5)$ & 51.1 & & & & \\
\hline \multirow[t]{2}{*}{ Mitotic count } & $\leq 5 / 50 \mathrm{HPF}$ & 65 (73.9) & 83.7 & 0.015 & 2.99 & $1.18-7.59$ & 0.021 \\
\hline & $>5 / 50 \mathrm{HPF}$ & $23(22.1)$ & 52.2 & & & & \\
\hline \multirow[t]{2}{*}{ Serosal involvement ${ }^{\ddagger}$} & Positive & $10(11.3)$ & 85.7 & 0.24 & & & \\
\hline & Negative & 75 (85.2) & 72.8 & & & & \\
\hline \multirow[t]{2}{*}{ Necrosis $^{\ddagger}$} & Positive & $25(28.4)$ & 68.5 & 0.236 & & & \\
\hline & Negative & $59(67.0)$ & 82.4 & & & & \\
\hline \multirow[t]{2}{*}{ Other cancer ${ }^{\ddagger}$} & Combined & $6(6.8)$ & 80 & 0.659 & & & \\
\hline & Not combined & 79 (89.8) & 73.9 & & & & \\
\hline
\end{tabular}

${ }^{*} P$-values were calculated with the Log-rank test.

${ }^{\dagger} P$-values were calculated with the Cox regression hazard model.

${ }^{\ddagger}$ Available cases only.

RFS, recurrence-free survival; HR, hazard ratio; HPF, high-power field.

the modified NIH criteria is more strongly associated with tumor recurrence after surgical resection than the very lowand low-risk groups (Fig. 1).

\section{Prognostic Factors after Curative Resection}

Many variables were evaluated to determine the association of the prognostic factors for GIST recurrence (Table 4). The tumor size $(P=0.05)$ and mitotic count $(P=0.015)$ were significantly associated with the risk of tumor recurrence with univariate analysis, whereas the presence of serosal involvement, presence of tumor necrosis, and combination with other cancers such as early or advanced gastric cancer were not statistically significant variables. With multivariate analysis, only mitotic count $(P=0.021)$ was significantly associated with prognosis. Although the difference was not statistically significant because of the small population of patients with GISTs of the colorectum, the 5 -year RFS was $73.9 \%$ and $50.0 \%$ in patients with GISTs in the small intestine and colorectum, respectively (Table 4).

\section{DISCUSSION}

The present study confirmed that the high-risk group according to the modified NIH criteria significantly experienced more recurrence than the other groups of patients with GISTs in the small intestine and colorectum in Korea. Complete surgical resection remains the mainstay of treatment for patients with localized GISTs, but many patients have a substantial risk of recurrence in the liver, peritoneum, or both. Clinical outcomes for patients with recurrence were dismal, and conventional chemotherapy and radiation therapy achieved a significant response only in $<10 \%$ of patients.

Imatinib, a tyrosine-kinase inhibitor, prolonged RFS after surgery for GIST in several studies and was approved as an adjuvant therapy for operable GISTs. ${ }^{14,20}$ In the National Comprehensive Cancer Network (NCCN) guidelines published in 2010, adjuvant imatinib therapy was highly recommended for patients with intermediate- to high-risk GISTs. ${ }^{10}$ In addition, adjuvant imatinib was proposed as an option for those patients with a substantial risk of relapse in the European Society for Medical Oncology (ESMO) guidelines. ${ }^{21}$ The Scandinavian Sarcoma Group (SSG) XVIII trial established that at least 36 months of adjuvant imatinib treatment had improved RFS and overall survival for patients with high-risk GISTs. ${ }^{22}$ Thus, 3 years of imatinib treatment became a standard adjuvant therapy for patients with high-risk GISTs in the NCCN guidelines that were updated in $2012 .{ }^{12}$

Until the ESMO guideline was published, "intermediate-tohigh risk" and "substantial risk" were not clearly defined. In the SSG XVIII trial, "high risk" was defined as tumor diameter $>5.0 \mathrm{~cm}$ and mitotic count $>5 / 50 \mathrm{HPF}$; tumor diameter $>10.0$ 
cm; mitotic count >10/50 HPF; or tumors ruptured into the peritoneal cavity. ${ }^{22}$ This definition of "high risk" is quite similar to that of the modified NIH criteria. Several studies were conducted to validate the risk stratification schemes, and the modified NIH criteria were identified as the most reliable tool for assessing prognosis of GIST as a result, especially for screening a single high-risk group. ${ }^{17,18}$

The present study corroborated a prognostic significance of the modified NIH criteria, which can be used effectively to determine whether individual patients need adjuvant imatinib therapy. As expected, both the very low- and lowrisk categories had a favorable prognosis under the original NIH or modified NIH risk classification. Only 4 patients experienced recurrence among 50 patients in the very lowand low-risk groups. According to the modified NIH criteria, all 22 patients with intermediate risk under the original NIH criteria were reclassified to high risk. All 6 patients who experienced tumor recurrence were reclassified as high-risk patients. Thus, postoperative surveillance for recurrence without adjuvant therapy can be recommended to the very low-, low-, and intermediate-risk patients according to the modified NIH criteria, considering the potential toxicities and financial cost of the imatinib treatment. However, recurrence also can occur in the very low-, low-, and intermediate-risk patients, so regular follow-up with radiographic examination for recurrence surveillance is strongly recommended. ${ }^{21}$

The 5-year RFS rates of very low/low-, intermediate-, and high-risk patients were inferior to those of a published metaanalysis, which were $91.0 \%, 69.0 \%$, and $43.0 \%$, respectively. ${ }^{21}$ This result may be related to the poor prognosis of GISTs in the small intestine and colorectum.

Recurrence in the high-risk group was uniformly distributed during the follow-up period, and this study proved the necessity of adjuvant therapy in that group. According to the Kaplan-Meier curves of the 5-year RFS, it may appear that the original NIH criteria are a better stratification system than the modified NIH criteria. Actually, the recurrence rates were statistically significantly different between the very low/low- $(8.0 \%, 4 / 50)$, intermediate- $(27.3 \%, 6 / 22)$, and highrisk $(50.0 \%, 8 / 16)$ categories according to the original NIH criteria during the follow-up period (median follow-up: 3.40 years, $P=0.036$ ). However, the most important aspect of the risk stratification system is to provide clinically useful standards. The main concern for patients with resectable GISTs is how to determine the patients who will need adjuvant chemotherapy after surgery. As previously described, the modified NIH criteria can determine the single most highrisk patient group for recurrence.
This study has several limitations including its singlecenter, retrospective design. In addition, recurrence developed only in $18 / 88$ patients (20.5\%), which was less than in previous studies and might be related to the small proportion of the high-risk group. In multivariate analysis, the mitotic count was a significant prognostic factor, but the primary tumor size was not, which might be related to the small proportion of patients with recurrence. Although the modified NIH criteria can distinguish the single high-risk patient group for consideration of adjuvant imatinib therapy, it cannot discriminate risk of recurrence between the very low-, low-, and intermediate-risk groups and cannot provide a quantifiable risk of recurrence for individual patients.

Accordingly, the modified NIH criteria predicted the recurrence of GIST after curative resection well, especially in the high-risk patients. Large population-based studies are needed to prove the relationship between recurrence and known prognostic factors in patients with GISTs in the small intestine and colorectum. Further studies of additional variables to predict the recurrence more precisely in order to individualize management are warranted.

\section{REFERENCES}

1. Miettinen M, Lasota J. Gastrointestinal stromal tumors: review on morphology, molecular pathology, prognosis, and differential diagnosis. Arch Pathol Lab Med 2006;130:1466-1478.

2. Hirota S, Isozaki K, Moriyama Y, et al. Gain-of-function mutations of c-kit in human gastrointestinal stromal tumors. Science 1998;279:577-580.

3. Fletcher CD, Berman JJ, Corless C, et al. Diagnosis of gastrointestinal stromal tumors: a consensus approach. Hum Pathol 2002;33:459-465.

4. Miettinen M, Sobin LH, Lasota J. Gastrointestinal stromal tumors of the stomach: a clinicopathologic, immunohistochemical, and molecular genetic study of 1765 cases with long-term follow-up. Am J Surg Pathol 2005;29:52-68.

5. Miettinen M, Makhlouf H, Sobin LH, Lasota J. Gastrointestinal stromal tumors of the jejunum and ileum: a clinicopathologic, immunohistochemical, and molecular genetic study of 906 cases before imatinib with long-term follow-up. Am J Surg Pathol 2006;30:477-489.

6. Miettinen M, Lasota J. Gastrointestinal stromal tumors: pathology and prognosis at different sites. Semin Diagn Pathol 2006; 23:70-83.

7. Rutkowski P, Nowecki ZI, Michej W, et al. Risk criteria and prognostic factors for predicting recurrences after resection of primary gastrointestinal stromal tumor. Ann Surg Oncol 2007;14: 
2018-2027.

8. Joensuu H. Predicting recurrence-free survival after surgery for GIST. Lancet Oncol 2009;10:1025.

9. Joensuu H. Risk stratification of patients diagnosed with gastrointestinal stromal tumor. Hum Pathol 2008;39:1411-1419.

10. Demetri GD, von Mehren M, Antonescu CR, et al. NCCN Task Force report: update on the management of patients with gastrointestinal stromal tumors. J Natl Compr Canc Netw 2010; 8(Suppl 2):S1-S41.

11. Demetri GD, von Mehren M, Blanke CD, et al. Efficacy and safety of imatinib mesylate in advanced gastrointestinal stromal tumors. N Engl J Med 2002;347:472-480.

12. von Mehren M, Benjamin RS, Bui MM, et al. Soft tissue sarcoma, version 2.2012: featured updates to the NCCN guidelines. J Natl Compr Canc Netw 2012;10:951-960.

13. Reynoso D, Trent JC. Neoadjuvant and adjuvant imatinib treatment in gastrointestinal stromal tumor: current status and recent developments. Curr Opin Oncol 2010;22:330-335.

14. Dematteo RP, Ballman KV, Antonescu CR, et al. Adjuvant imatinib mesylate after resection of localised, primary gastrointestinal stromal tumour: a randomised, double-blind, placebocontrolled trial. Lancet 2009;373:1097-1104.

15. Hohenberger P. Adjuvant imatinib in GIST: a self-fulfilling prophecy, or more? Lancet 2009;373:1058-1060.

16. Gronchi A, Judson I, Nishida T, et al. Adjuvant treatment of GIST with imatinib: solid ground or still quicksand? A comment on behalf of the EORTC Soft Tissue and Bone Sarcoma Group, the Italian Sarcoma Group, the NCRI Sarcoma Clinical Studies Group (UK), the Japanese Study Group on GIST, the French Sarcoma Group and the Spanish Sarcoma Group (GEIS). Eur J Cancer 2009;45:1103-1106.

17. Joensuu H, Vehtari A, Riihimaki J, et al. Risk of recurrence of gastrointestinal stromal tumour after surgery: an analysis of pooled population-based cohorts. Lancet Oncol 2012;13:265274.

18. Rutkowski P, Bylina E, Wozniak A, et al. Validation of the Joensuu risk criteria for primary resectable gastrointestinal stromal tumour - the impact of tumour rupture on patient outcomes. Eur J Surg Oncol 2011;37:890-896.

19. Joensuu H. Adjuvant therapy for high-risk gastrointestinal stromal tumour: considerations for optimal management. Drugs 2012;72:1953-1963.

20. Eisenberg BL, Harris J, Blanke CD, et al. Phase II trial of neoadjuvant/adjuvant imatinib mesylate (IM) for advanced primary and metastatic/recurrent operable gastrointestinal stromal tumor (GIST): early results of RTOG 0132/ACRIN 6665. J Surg Oncol 2009;99:42-47.

21. Casali PG, Blay JY. Gastrointestinal stromal tumours: ESMO Clinical Practice Guidelines for diagnosis, treatment and followup. Ann Oncol 2010;21(Suppl 5):v98-v102.

22. Joensuu H, Eriksson M, Sundby Hall K, et al. One vs three years of adjuvant imatinib for operable gastrointestinal stromal tumor: a randomized trial. JAMA 2012;307:1265-1272. 